\title{
En quête de la méthode directe dans le fonds Ferdinand Brunot, réflexion épistémologique fondée sur un échec heuristique
}

In search of the direct method in the Ferdinand Brunot collection: an epistemological reflection based on a heuristic failure

Clémentine Rubio et Marc Debono

\section{OpenEdition \\ Journals}

\section{Édition électronique}

URL : https://journals.openedition.org/dhfles/7911

DOI : $10.4000 /$ dhfles.7911

ISSN : 2221-4038

\section{Éditeur}

Société Internationale pour l'Histoire du Français Langue Étrangère ou Seconde

\section{Édition imprimée}

Date de publication : 1 décembre 2020

Pagination : 203-218

ISSN : 0992-7654

Référence électronique

Clémentine Rubio et Marc Debono, «En quête de la méthode directe dans le fonds Ferdinand Brunot réflexion épistémologique fondée sur un échec heuristique », Documents pour l'histoire du français langue étrangère ou seconde [En ligne], 64-65 | 2020, mis en ligne le 24 février 2021, consulté le 25 mars 2023. URL : http://journals.openedition.org/dhfles/7911; DOI : https://doi.org/10.4000/dhfles.7911

Ce document a été généré automatiquement le 25 mars 2023.

Tous droits réservés 


\title{
En quête de la méthode directe dans le fonds Ferdinand Brunot, réflexion épistémologique fondée sur un échec heuristique ${ }^{1}$
}

\author{
In search of the direct method in the Ferdinand Brunot collection: an \\ epistemological reflection based on a heuristic failure
}

Clémentine Rubio et Marc Debono

\section{Introduction : Ferdinand Brunot, un méthodologue contemporain de la méthode directe}

1 La réflexion méthodologique et épistémologique proposée dans cet article fut amorcée à l'occasion d'une communication lors du colloque de la SIHFLES, portant sur la méthode directe, organisé à Grenade en 2019. Cette recherche exploratoire a été menée à partir de documents présents dans le "fonds Brunot» de l'université de Tours. La bibliothèque de l'université de Tours détient en effet depuis 50 ans une importante collection d'ouvrages issue de la bibliothèque personnelle de Ferdinand Brunot ${ }^{2}$.

Il semble utile en préambule de souligner en quoi Ferdinand Brunot nous est apparu comme un personnage digne d'intérêt pour réfléchir à la méthode directe - méthode qui lui fut contemporaine - à travers l'exploration de sa bibliothèque personnelle (1.), exploration qui aura elle-même débouché sur une réflexion épistémologique quant à la manière de faire de l'histoire du FLE/S (2.).

3 Ferdinand Brunot, né en 1860, est un grammairien et historien de la langue qui a obtenu en 1900 la première chaire d'histoire de la Langue Française à la Sorbonne. Ce personnage historique est particulièrement intéressant du fait du constant dialogue entre sa passion pour la grammaire historique et la question de l'enseignement du français (en particulier à des étrangere.s). Un linguiste donc, mais qui s'est intéressé de 
près à la pédagogie du français et à sa diffusion internationale à travers les nombreuses fonctions qu'il a occupées: il a en particulier dirigé des cours d'été de l'Alliance Française pendant 10 ans de 1894 à 1904 et fut le fondateur en 1920 de l'École de préparation des professeurs de français à l'étranger (devenu l'Institut de préparation des professeurs de français à l'étranger) ${ }^{3}$.

Beaucoup de choses ont été écrites quant à la pensée méthodologique de Brunot, notamment à travers les travaux menés au sein de la SIHFLES. Essentiellement appliquées à ce que l'on appellerait aujourd'hui le "français langue maternelle », les inspirations méthodologiques du philologue ont été largement détaillées dans les écrits de René Lagane (1972), de Jean-Claude Chevalier (1990 et 1991), de Ludo Melis \& Pierre Swiggers (1992), d'Henri Besse (1995) ou encore de Jean-Louis Chiss (1995). Ce que Brunot appelle "méthodologie spéciale » de la langue française est principalement exposée dans L'enseignement de la langue française, ce qu'il est, ce qu'il devrait être dans l'enseignement primaire en 1909 et dans La pensée et la Langue. Méthode, principes et plan d'une théorie nouvelle du langage appliquée au français en 1922. Les grands principes de cette nouvelle méthodologie, élaborée au tournant du siècle dernier sont précisément résumés par Besse dans un article de 1995 intitulé « Ferdinand Brunot, méthodologue de la langue française", avec notamment une réflexion poussée autour de la grammaire et son enseignement. Il s'agit en particulier pour Brunot de subordonner l'enseignement de la grammaire à celui de la langue, véritable « combat »- empruntant bien souvent les voies de la polémique - contre les pesanteurs grammaticalisantes de la tradition pédagogique. Point également original pour l'époque, les doutes exprimés sur les méthodologies universalistes, Brunot souhaitant introduire "une souplesse de doctrines qui n'existe pas et qu'exigent cependant la diversité extrême et la mobilité incessante du langage », projet « délicat et complexe » qui ne peut pas déboucher sur une "méthode infaillible et universelle pour enseigner à tous la langue française " (conférence de 1911, cité par Besse, 1995). Malgré ses originalités, force est de constater, avec les historien.nes-didacticien.nes qui ont étudié Brunot et sa réception, que sa "méthode spéciale » ne rencontrera pas un franc succès. Les raisons en sont explorées par plusieurs chercheur.es : trop complexe, trop « en avance », parfois jugée " évasive » sur des points précis de langue également, etc. Il n'en reste pas moins que cette élaboration didactique est rétrospectivement assez surprenante quand on considère aujourd'hui l'intérêt très relatif des «linguistes" ou historien.nes de la langue pour les questions didactiques.

5 La proximité de la «méthode spéciale » de Brunot avec la méthode directe nous a en tout cas amené.es à penser que sa bibliothèque personnelle pouvait révéler des affinités intéressantes entre les deux constructions méthodologiques contemporaines et ainsi éclairer l'histoire de la méthode directe.

\section{Parcours d'un échec et de son intérêt heuristique}

6 Ainsi, la perspective que nous avions initialement adoptée était une perspective en miroir. Nous souhaitions interroger d'une part la manière dont on pourrait travailler sur la méthode directe à partir du fonds Brunot, et d'autre part, à l'inverse la manière dont on pourrait travailler sur le fonds Brunot à partir de l'« entrée » méthode directe, pour en tirer une réflexion méthodologique et épistémologique ( $c f$. infra). Autrement dit, nous nous proposions de travailler à partir de deux entrées. La première était celle 
d'une "archéologie de la méthode directe », partant du constat de Besse selon lequel « les techniques les plus caractéristiques de la méthode directe [avaient] été mises en pratique, décrites voire théorisées des siècles avant que ses tenants ne la promeuvent» (Besse 2012). Nous nous sommes demandé si le fonds Brunot donnait à voir des théorisations précoces de la méthode directe ou d'une réflexion montante qui laisserait présager de l'élaboration de cette méthode, mais aussi si le fonds donnait à lire les inspirations de Brunot dans l'élaboration de sa propre "méthode spéciale", contemporaine de la méthode directe, et donc les liens affinitaires entre les deux "méthodes» (cf. infra: Brunot écrit lui-même s'être largement inspiré de la méthode directe). La seconde entrée était davantage épistémologique et visait à poser des questions sur la manière de mener une recherche historique, en particulier à partir d'un fonds documentaire personnel: l'entrée «Méthode directe » représentant une occasion de nous interroger sur ce qui teinte notre regard de chercheur.es (nos expériences, nos projets de lecture). Travailler sur le fonds à partir d'un projet (autour de la méthode directe) nous permettait donc de poser des questions quant à la manière d'appréhender des sources, des archives, des traces, etc. pour faire l'histoire d'un champ, le FLE/S en l'occurrence. Cette réflexion à dominante épistémologique étant pour nous une manière de contribuer à l'écriture de cette histoire disciplinaire.

7 Si cette seconde entrée s'est avérée riche d'enseignements ( $c f$. infra), il s'est rapidement avéré que la première était une impasse, dont nous n'avions pas entièrement conscience en nous lançant dans l'aventure : le fonds Brunot ne dit pas grand-chose ni de l'arrivée de la méthode directe, ni de l'élaboration méthodologique de Brunot, telle qu'elle apparaît dans ses différents ouvrages (La pensée et la langue de 1922 et Méthode de Langue Française de 1905).

8 Constatant cette impasse, différents choix s'offraient à nous. Un premier choix aurait pu être celui de considérer que de ne « rien trouver » était synonyme d'échec et de ne pas en tirer d'interprétation. La question est alors de savoir si l'on peut ne rien trouver, en particulier en Sciences Humaines et Sociales ${ }^{4}$, ou encore si le fait de ne pas trouver l'objet initial d'une recherche est synonyme d'une mauvaise recherche.

9 Un second choix, celui qui donne lieu à cet article, a été celui de travailler sur les différents éléments qui permettent de comprendre l'impasse dans laquelle nous nous sommes trouvé.es et de les (re)-problématiser. Cette problématisation nous a permis de formuler trois pistes pouvant expliquer notre difficulté/l'impossibilité relative de trouver des traces ${ }^{5}$ de la méthode directe dans le fonds Brunot:

10 - la première concerne le fonds en lui-même, son contenu et son organisation, ainsi que ce qu'un fonds peut nous dire d'une pensée ;

11 - la seconde réponse vient de la manière dont Brunot a élaboré sa pensée didactique dans une démarche profondément empirique ;

12 - enfin la dernière réponse remonte à notre projet initial, et ce qu'il implique en termes de conception de l'histoire. 


\section{Des pistes d'exploration du fonds Brunot semées d'obstacles}

\section{À la recherche de traces d'émergence de la méthode directe dans un fonds documentaire personnel}

Le fait qu'il n'y ait pas de traces de la méthode directe et de l'élaboration de la pensée didactique de Brunot, ou que celles-ci ne soient pas facilement identifiables, peut selon nous tenir dans un premier temps d'analyse à deux facteurs : le contenu du fonds et son mode de répertoriage.

Dans le fonds se trouvent quelques 14000 documents référencés en catalogue sans organisation thématique, chronologique ou par mots clés, ce qui rend difficile un quelconque repérage spécifique à un sujet. Si des thèmes sont vaguement regroupés (les grammaires se trouvent par exemple presque toutes sur les mêmes rayonnages), cela reste marginal. Ces documents sont sous des formats différents, et sont rédigés dans des langues différentes. On trouve des ouvrages politiques, juridiques, de médecine, de philosophie, de religion, de littérature, des controverses et débats et de nombreux ouvrages sur les langues avec en particulier d'innombrables grammaires et dictionnaires.

Le fonds Brunot porte bien son nom, dont Arlette Farge (1989: 10) rappelle la signification métaphorique : des documents qui sont en définitive « reliés ensemble par le seul fait d'avoir un jour été donnés ou légués par un particulier qui en avait la propriété » (ibid.), dont l'exploration relève en effet d'une plongée dans l'obscurité insondable des fonds marins.

16 Le mode de répertoriage joue ainsi un rôle important et le fait qu'aucune classification thématique n'existe à ce jour pour le fonds Brunot a participé de la difficulté à trouver des traces de la méthode directe (et, plus largement, toute trace dans le cadre d'une recherche sur une thématique précise). Le travail de repérage commencé sur ce fonds ${ }^{6}$ consiste d'ailleurs à classifier, à proposer des mots-clés pour rendre ce fonds accessible à des chercheur.es. Il ne faut cependant pas perdre de vue que tout classement est aussi une réduction des documents du fonds ainsi appréhendés, un enfermement dans une catégorie qui masque la diversité des lectures que l'on pourrait en faire. Bien que celui ou celle qui classe réfléchisse à ce qui peut intéresser les chercheur.es, il ou elle pense à la fois avec ses propres catégories, ses propres grilles de lecture mais aussi à partir des catégories qui lui sont imposées par l'archivistique (mots clés, cotes, etc.). Les catégories finalement proposées seront autant des entrées qui s'ouvrent sur un document qu'une manière de les enfermer? .

\section{Que peut-on comprendre à partir d'un fonds personnel ?}

17 L'accès au fonds Brunot, à la bibliothèque personnelle de ce personnage, est précieux, mais que peut-il nous apprendre de l'homme et de son rapport à son temps, et en particulier à la méthode directe?

Ce fonds présente un intérêt scientifique évident: il est le témoignage d'un recueil d'ouvrages d'une époque, chez un homme passionné par la grammaire historique, par l'histoire de la langue française et par son enseignement. Il s'agit de surcroît de la 
collection d'un homme avec des moyens d'acquisition que l'on imagine importants, notamment du fait des postes importants qu'il a occupés. On peut aussi imaginer qu'il recevait des sollicitations de lectures, et un certain nombre d'ouvrages en témoignent par les petites notes personnelles qu'ils contiennent, adressées par leurs auteurs à Brunot. Ce fonds est ainsi à lui seul un inventaire d'une grande partie de ce qui s'est écrit à l'époque de Brunot (et bien avant) sur le français (en particulier sur la grammaire), y compris les débats suscités par un certain nombre d'ouvrages. En ce sens, cette bibliothèque établie en tant que "fonds ", dans une démarche patrimoniale, constitue un ensemble de ressources, notamment pour le champ de l'histoire de la diffusion du français et de l'enseignement du français langue étrangère et seconde.

Pourtant, si les ouvrages en provenance de la bibliothèque de Brunot reflètent, dans une certaine mesure, les divers domaines d'intérêts de Brunot, il est particulièrement difficile d'en jauger les usages, ou l'influence qu'aurait pu avoir tel ou tel ouvrage sur sa pensée et sur ses liens avec l'élaboration de la méthode directe. Une recherche menée sur la collection d'un tel bibliophile ne peut ainsi passer à côté d'un certain nombre de questions concernant les nombreux ouvrages conservés: dans ce fonds gigantesque, quels sont les ouvrages qu'il a reçus et de qui ? Quels sont ceux qu'il a lus ? Et aussi, quels sont les ouvrages absents, ceux qu'il a donnés, ceux perdus en route ou que sa famille a conservés ? Et plus avant, qu'a-t-il pensé de ces ouvrages? Autrement dit, il est extrêmement difficile de savoir comment les ouvrages contenus dans le fonds ont pesé sur sa pensée pour contraster la méthode directe et sa propre méthode.

Comme toute aventure intellectuelle, la pensée de Brunot s'est par ailleurs nécessairement inscrite dans un réseau, dans des échanges et par le biais de débats, de conférences, de courriers non accessibles, non conservés ou non dépouillés.

Ces «absences" ne sont pas spécifiques à la recherche sur un fonds. C'est un thème bien connu des historien.nes et de la recherche à partir d'archives. Les questions que ces absences posent permettent de mettre en avant des enjeux méthodologiques et épistémologiques sur les manières de faire l'histoire du français langue étrangère (sur lesquelles nous reviendrons plus loin).

Pour les comprendre, on peut aussi considérer que ces absences, ces non-traces ont quelque chose à nous apprendre de l'élaboration de la méthode directe : si celle-ci n'est que peu appréhensible par le biais de ce fonds personnel, c'est peut-être qu'elle s'est faite aussi en partie hors des livres?

\section{Une élaboration didactique « empirique » et un fonds silencieux}

$23 \mathrm{Si}$, comme dit précédemment, il nous apparaît inutile ici de revenir plus en détail sur la pensée méthodologique de Brunot, ce que l'on peut utilement rappeler, c'est que la "méthodologie spéciale» de la langue française qu'il développe a été largement inspirée par l'émergence à l'orée du $\mathrm{XX}^{\mathrm{e}}$ siècle, dans le domaine des langues vivantes, de la méthode directe, dont il reconnaît toute la valeur :

Au dernier congrès des langues vivantes que j'avais l'honneur de présider, personne ne contestait la valeur de la méthode directe, la méthode maternelle en somme (Brunot 1911: 50 ; cité par Besse $1995: 47)^{8}$. préparation de ce travail, était la suivante: peut-on trouver des traces de cette inspiration liée à une attention particulière portée à la méthode directe, dans la 
bibliothèque personnelle de Brunot ? Cela parait fortement compromis lorsque celui-ci affirme lui-même que sa familiarisation avec les pratiques directes d'enseignement s'est principalement faite "par empirisme ", au contact des professeur.es de langues vivantes, notamment dans le cadre des cours d'été de l'Alliance française pendant ces dix années de direction de 1894 à 1904 :

C'est par empirisme, je l'avoue, que j'ai été amené peu à peu à la conviction qu'il fallait renoncer aux anciens cadres (1920:163).

Je me flatte que les professeurs de langues vivantes accepteront presque sans résistance une révolution [celle de La Pensée et la Langue] qu'ils ont préparée. Leur pratique appelait ma théorie $(1921: 37)^{9}$.

Ces propos montrent, rétrospectivement, l'impasse (toute relative comme nous allons essayer de le montrer) d'une partie de notre projet initial, celle qui consistait à faire l'archéologie de la méthodologie directement à travers le fonds détenu par la bibliothèque universitaire de Tours : nous touchons ici aux limites (évoquées supra) de l'exploration d'une bibliothèque pour connaître l'homme ou une partie de la vie intellectuelle de celui-ci. Nous aurions certes pu considérer l'empirisme conscientisé de cette élaboration didactique avant de nous lancer dans la fouille de ce fonds, mais il est tout aussi possible de considérer, positivement, que son caractère infructueux confirme, d'une autre manière, ces dires du Brunot méthodologue : son élaboration méthodologique est inspirée d'une méthode qu'il a d'abord vue à l'œuvre dans les cours de l'Alliance française ${ }^{10}$. On pourrait bien entendu trouver quelques ouvrages de sa bibliothèque allant dans le sens d'une telle évolution, mais on constate surtout que ces ouvrages sont en général postérieurs à cette décennie 1894-1904 durant laquelle Brunot a été au contact direct des praticien.nes de l'Alliance française, que l'on peut considérer avec Besse comme l'époque de son «tournant méthodologique» quant à l'enseignement de la langue française. Nous n'avons trouvé aucun document susceptible d'éclairer ce tournant méthodologique... Rebondissons néanmoins après cette probable déception de l'horizon d'attente de notre lecteur : finalement, peut-on vraiment dire que nous n'avons « rien trouvé »?

\section{Tirer parti d'un échec : des projets de parcours interprétatifs}

Ouvrons dès à présent ce qui sera l'objet d'un point à venir, l'inscription de toute recherche dans des projets et des histoires de chercheur.es, qui ne devraient être écartés de la recherche elle-même. Ainsi, une manière de comprendre le relatif échec de cette recherche consiste à examiner les motivations premières de celle-ci et nos inscriptions respectives dans des questionnements épistémologiques et éthiques.

\section{Visibilisation du fonds Brunot}

Notre projet initial n'était pas uniquement, voire pas prioritairement, de trouver une élaboration méthodologique présageant de l'émergence de la méthode directe chez Brunot. Nous ne partions ni d'une recherche sur la méthode directe qui nous aurait mené.es vers le fonds Brunot (nous ne nous serions pas intéressé.es à Brunot pour parler de la MD si sa bibliothèque n'avait été à Tours), ni moins encore d'une certitude, ou d'une recherche déjà presque close dont nous n'aurions eu qu'à tirer une analyse. 
Presque à l'inverse, nous nous sommes saisi.es de l'occasion d'un colloque scientifique portant sur la méthode directe pour rendre plus visible, faire connaitre ce fonds peu exploité et pour l'explorer avec une « entrée d'exploration » précise.

Ainsi, si l'objectif de comprendre l'élaboration de la méthode directe via une bibliothèque n'est pas atteint, celui de faire connaître le fonds et ce qu'il peut apporter à la recherche en histoire du français langue étrangère et seconde, l'est davantage, de manière un peu détournée nous en convenons.

\section{L'occasion de poursuivre une réflexion épistémologique sur la place de l'histoire en DDL}

29 Une autre partie de notre projet était de porter à notre tour, en tant que chercheur.es/ siflhésien.nes, une démarche historicisante en DDL qui nous semble pouvoir salutairement (re)jouer le rôle que certains de ses promoteurs initiaux lui assignaient, notamment au sein de la SIHFLES au moment de sa constitution : aller à l'encontre des «niaiseries positivistes » (pour reprendre la formule polémique de Clavères 1990), relativiser la prégnance dans le champ didactique des séduisants chants de l'innovation permanente, portés par des formes d'illusions technicistes. Le défi pour l'histoire de la DDL est de taille, et il nous semble que pour le relever, il faut aussi accepter de faire une histoire " non positiviste ", qui reconnaît et assume éthiquement les projets de ceux qui la font. En un mot: faire de l'histoire nous semble toujours plus important à mesure que la DDL oublie son passé pour des formes à notre sens délétères de présentisme/ futurisme, mais à condition de ne pas verser dans une histoire qui emprunterait les voies méthodologiques et épistémologiques de ce qu'elle s'emploie à dénoncer par ailleurs. Ce dernier point mérite sans doute un débat collectif approfondi, et les propos qui suivent n'ont d'autre ambition que de contribuer à modestement l'initier.

Partons d'une illustration, avec le projet interprétatif assumé par Chiss en ouverture de son article de 1995 sur le rapport de Bally et Brunot à la DDL (« A partir de Bally et Brunot: la langue française, les savants et les pédagogues»), dans lequel il propose d'« explorer les modalités de l'intérêt des linguistes français et francophones pour l'enseignement/apprentissage de la langue maternelle et des langues étrangères à la charnière $\mathrm{XIX} / \mathrm{XX}^{\mathrm{e}}$ siècles et à faire percevoir (par contraste et/ou continuité) la situation de la communauté savante des linguistes aujourd'hui vis-à-vis de la constitution du domaine de la didactique des langues" (Chiss 1995: 20; nos italiques). On ne saurait exposer plus clairement un projet de " parcours interprétatif » d'œuvres d'auteur.es du passé. Quand on connaît un peu le contexte français de développement de la didactique des langues/ du FLE au sein des sciences du langage dans les années 1970-80, développement marqué par les luttes de pouvoirs liées à l'autonomisation par rapport à une linguistique tutélaire et à l'affirmation d'une identité disciplinaire (luttes qui ont alors généré des tensions entre "linguistes " et "didacticien.nes ", encore fortes en 1995 et toujours perceptibles aujourd'hui), on mesure bien le sens de ce propos liminaire. Il peut être résumé de manière fort triviale, à l'aide d'une formule certes galvaudée, mais néanmoins toujours éclairante de bien des propos d'historiens : chercher dans le passé des moyens d'éclairer les enjeux du présent. L'exploration d'un corpus historique est donc fondamentalement biaisée : mais, dans la conception que nous avons du travail historique, ce "biais » est pour nous non-problématique, car il relève d'un horizon d'interprétation et constitue par là-même le «fondement de la rétrodiction » de 
l'historien.ne évoqué par Paul Veyne (cf. citation infra). Proposer la lecture d'un corpus historique avec un projet précis n'est pas un problème en soi, si celui-ci est assumé en tant que tel, avec une tentative d'explicitation de ce qui le motive et le fonde ${ }^{11} . \mathrm{Si}$, autrement dit, on rompt avec la « règle du jeu » si clairement exposée par Veyne dans Comment on écrit l'histoire (1971) :

Car la seconde limite de l'objectivité [...] est la variété des expériences personnelles, qui sont malaisément transmissibles. Deux historiens des religions ne seront pas d'accord sur le «symbolisme funéraire romain », parce que l'un a l'expérience des inscriptions antiques, des pèlerinages bretons, de la dévotion napolitaine et qu'il a lu Le Bras, pendant que l'autre s'est fait une philosophie religieuse à partir des textes antiques, de sa propre foi et de sainte Thérèse ; la règle du jeu étant qu'on ne cherche jamais à expliciter le contenu des expériences qui sont le fondement de la rétrodiction, il ne leur restera plus qu'à s'accuser mutuellement de manquer de sensibilité religieuse, ce qui ne veut rien dire, mais se pardonne difficilement (Veyne 1971:212; nos italiques).

Ces « expériences personnelles [...] malaisément transmissibles » fondent pourtant des projets de lectures: on comprend toujours (un corpus de textes historiques, une archive, un fonds, etc.) «à partir de » (ces expériences) et « en vue de » (avec un/des projets). Ce que d'aucuns appellent des «biais» - dans un autre univers épistémologique - ne sont pas ici considérés comme des obstacles, mais comme la condition de la compréhension de ce qui est appréhendé (la pensée méthodologique de Brunot à travers sa bibliothèque personnelle par exemple), mais constitue la condition même de cette compréhension. En l'occurrence, dans ce travail, notre compréhension $\mathrm{du}$ fonds Brunot était fondée sur un double projet de donner plus de visibilité à une bibliothèque personnelle méconnue et de réflexion théorique sur la manière de faire l'histoire.

Le philosophe allemand Hans-Georg Gadamer (1900-2002) propose comme pierre angulaire de son herméneutique l'idée de précompréhension (Vorverständnis) : toute compréhension implique une "structure d'anticipation " (Vorstruktur: Gadamer 1996), un processus de précompréhension inévitable, qui s'enracine dans l'expérience et les projets de l'interprète, lesquels donnent corps à ses préjugés. Loin d'être une force négative, déformante, le préjugé constitue chez Gadamer une condition positive de la connaissance. Pour comprendre et se comprendre, il est donc nécessaire de partir de ceux-ci: "Ce n'est qu'en reconnaissant ainsi que toute compréhension relève essentiellement $\mathrm{du}$ préjugé que l'on prend toute la mesure du problème herméneutique » (Gadamer $1996: 291$ ).

\section{En guise de conclusion}

En définitive, en partant de ce «fond » épistémologique, il s'agit pour nous de proposer une autre mobilisation de l'histoire en DDL : après avoir servi à asseoir la légitimité d'une discipline en construction, autonomisation, consolidation ${ }^{12}$, après une « documentation » historique nécessaire du champ, peut-être est-il temps de réfléchir collectivement à la manière de penser et faire cette histoire, et ainsi approfondir certaines des questions initiales posées au sein de la SIHFLES ? Il serait par exemple intéressant de débattre au sein de la communauté sihflésienne du rôle politique que peut jouer aujourd'hui l'histoire en DDL, aux côtés (et en synergie avec) du - toujours 
essentiel - rôle de documentation et de légitimation que notre société savante assume remarquablement depuis sa création.

Pour que l'histoire de la DDL ne reste pas un « hobby d'érudits » vaguement « tabou » ${ }^{13}$, pour reprendre les mots même du premier président de la SIHFLES (Coste 1990: 8), peut-on, collectivement, lui donner cette dimension politique, en intégrant historiquement les débats contemporains, avec des projets qui peuvent être divers et variés, à envisager en tant que tels pour qu'ils puissent être débattus?

\section{BIBLIOGRAPHIE}

BESSE, Henri (1995). « Ferdinand Brunot, méthodologue de l'enseignement de la langue française ». Histoire Épistémologie Langage, tome 17, fascicule 1. En ligne : [https://www.persee.fr/ doc/hel_0750-8069_1995_num_17_1_2402].

BESSE, Henri (2012). « Éléments pour une 'archéologie' de la méthode directe ». Documents pour l'histoire du français langue étrangère ou seconde, 49, 11-30.

BESSE, Henri (2016). « Cinq questions sur l'étude historique des 'innovations pédagogiques' en didactique des langues étrangères ou secondes (L2) ». Documents pour l'histoire du français langue étrangère ou seconde, 57, 11-31.

CHEVALIER, Jean-Claude (1990). «Syntaxe et sémantique en grammaire. Histoire d'une méprise : F. Brunot et Ch. Bally ». In R. Liver, I. Werlen, \& P. Wunderli, (éds), Sprachtheorie und Theorie der Sprachwissenschaft. Geschichte und Perspektiven. Festschrift für Rudolf Engler zum 60. Geburtstag. Tübingen : Narr, 95-107.

CHEVAliER, Jean-Claude (1991). « Ferdinand Brunot, La Pensée et la Langue ». In Hélène Huot (éd.), La grammaire française entre comparatisme et structuralisme (1870-1960). Paris : Colin, 73-114.

CHEVALIER, Jean-Claude (2010). «Ferdinand Brunot et les débuts de l'École de préparation des professeurs de français à l'étranger ». Documents pour l'histoire du français langue étrangère ou seconde, 44, 15-27.

CHISS, Jean-Louis (1995). « À partir de Bally et Brunot : la langue française, les savants et les pédagogues ». Histoire Épistémologie Langage, tome XVII, fascicule 1. Théories du langage et enseignement des langues (fin du XIX $X^{e}$ siècle/début du XX siècle) 19-40. En ligne : [https:// www.persee.fr/doc/hel_0750-8069_1995_num_17_1_2401].

CLAVÈRES, Marie-Hélène (1990). « Portrait de Thésée en peaux de chèvres ». Études de linguistique appliquée, 78, 125-133.

COSTE, Daniel (1990). « Pour ne plus se raconter d'histoires ». Études de linguistique appliquée, 78, 5-17.

DEBONO, Marc (2016). «Que fait l'innovation à la didactique des langues ? Éléments d'histoire notionnelle pour une réflexion (très) actuelle ». Documents pour l'Histoire du français langue étrangère ou seconde, 57, 29-51.

FARGE, Arlette (1989). Le goût de l'archive, Paris : Seuil. 
GADAMER, Hans-Georg (1996 [1976]). Vérité et Méthode, trad. P. Fruchon, Paris : Seuil.

LAGANE, René (1972). « Science linguistique et normativité : le cas de Ferdinand Brunot ». Langue française, 16, 88-98. En ligne : [https://www.persee.fr/doc/lfr_0023-8368_1972_num_16_1_5706].

MÉLIS, Ludo \& SWIGGERS, Pierre (1992). « Ferdinand Brunot contre la sclérose de la grammaire

scolaire ». Cahiers Ferdinand de Saussure, 46, 143-158.

PORCHER, Louis (1984). « Didactique historique ». In Daniel Coste, (dir.). Aspects d'une politique de diffusion du français langue étrangère depuis 1945. Matériaux pour une histoire. Paris : Hatier, 250-254.

RICœUR, Paul (1955). Histoire et vérité, Paris : Seuil

VeYNE, Paul (1996). Comment on écrit l'histoire, Paris : Seuil.

\section{NOTES}

1. Ce texte a bénéficié des relectures avisées d'Emmanuelle Huver et de Véronique Castellotti, que nous remercions. Nous restons cependant les seul.es responsables des propos qui y sont tenus.

2. Ce fonds a été racheté au fils de Ferdinand Brunot par l'université d'Orléans, puis récupéré à Tours.

3. Éléments biographiques tirés de Besse 1995.

4. On pourrait étendre cette question à tous les domaines scientifiques. Le récent projet Gaffex, qui propose de publier les recherches n'ayant pas abouti, dans la mesure où elles permettent des recherches à succès par les questions qu'elles posent, est un bon exemple : «Afin d'éviter que les recherches scientifiques qui n'ont pas abouti tombent dans l'oubli, un chercheur monte avec d'autres une plate-forme qui publie les expériences ratées. Elle sera ouverte à tous » (30 septembre 2019, radio France Inter). En ligne : [https://www.franceinter.fr/emissions/l-esprit-dinitiative/l-esprit-d-initiative-30-septembre-2019].

5. Au sens que Ricœur reprend de Simiand: "l'histoire est une connaissance par traces » (Ricœur 1955 : 25), l'historien n'étant jamais devant le passé et ne pouvant qu'observer, au sens fort, des traces.

6. Projet Valoriser le fonds Ferdinand Brunot de l'Université de Tours démarré en 2017 et porté par C. Rubio.

7. Sur ces questions nous renvoyons à la conférence filmée Voyage dans un carton d'archives, Mathias Enard et Leyla Dakhli, RDV de l'Histoire du monde arabe, 2016. En ligne: [https:// www.youtube.com/watch?v=kmv1eqiJIeQ].

8. Besse note par ailleurs l'assimilation erronée par Brunot de la méthode «directe » à la méthode « maternelle ».

9. Propos tirés des deux articles de Brunot sur «Le renouvellement nécessaire des méthodes grammaticales », publiés successivement dans la Revue Universitaire, cités par Besse 1995 : 47 (les mises en italiques sont de notre fait).

10. Il s'agit bien entendu d'une hypothèse, à nuancer par d'autres hypothèses : en effet Brunot a pu mettre en avant l'empirisme de sa démarche pour différentes raisons : tactiques (le «terrain » comme preuve légitimante de la pertinence de sa méthodologie : tactique fréquente en DDL); ou encore $\mathrm{du}$ fait d'une séparation nette dans son esprit entre pratique (élaboration d'une méthodologie) et théorie (lectures, connaissances livresques), séparation que l'on sait contestable (les lectures de Brunot - présentes dans le fonds ou non - ont pu inspirer sa pensée didactique, même si elles portaient sur des sujets a priori éloignés). Merci à Emmanuelle Huver de nous avoir signalé ces aspects. 
11. Ce que nous essayons de faire dans ce texte, certainement de manière encore un peu maladroite.

12. "C'est en maîtrisant les transformations historiques que nous avons une chance de parvenir à gouverner notre avenir. Parce que notre didactique est aujourd'hui constituée, elle est maintenant appelée à s'interroger sur sa nature, c'est-à-dire sur ce qu'elle tient de conditions de sa naissance" proposait Louis Porcher comme programme à sa "didactique historique", ajoutant que « si elle se refusait à le faire, elle se refermerait seulement, comme une parenthèse » (Porcher $1984:$ 254).

13. Tabou lié au «présentisme » caractérisant largement la DDL depuis ses origines, couplée au «futurisme» de la fuite en avant de l'innovation perpétuelle: on préfèrerait ne pas trop aller voir ce qui s'est dit et fait, au risque de relativiser l'importance de ce qui a été " découvert ». Sur cette question de l'histoire de l'innovation en DDL, voir en particulier les contributions au numéro 57 (2016) de A.-C. Santos (dir), «Innovations pédagogiques dans l'enseignement des langues étrangères: perspective historique (XVI $-\mathrm{XXI}^{\mathrm{e}}$ siècles)». Documents pour l'histoire du français langue étrangère ou seconde, (notamment Besse : 11-31 et Debono : 33-56).

\section{RÉSUMÉS}

La bibliothèque de l'Université de Tours détient depuis 50 ans une importante collection d'ouvrages (près de 15000 documents) issue de la bibliothèque du célèbre linguiste et historien de la langue que fut Ferdinand Brunot. Après avoir souligné en quoi Brunot nous est apparu comme un personnage digne d'intérêt pour réfléchir à la méthode directe - méthode qui lui fut contemporaine - à travers l'exploration de sa bibliothèque personnelle, nous expliquerons en quoi l'échec relatif de cette exploration aura débouché sur une réflexion épistémologique quant à la manière de faire de l'histoire du FLE/S.

The Tours university library has held for 50 years an important collection of books (nearly 15,000 documents) from the private library of the famous linguist and language historian Ferdinand Brunot. After underlining the reasons why Brunot appeared as a character worthy of interest to study the direct method - a method which emerged during his lifetime - through the exploration of his personal library, we will explain how the relative failure of this exploration has led to an epistemological reflection on the history of French as a Foreign/Second Language.

\section{INDEX}

Mots-clés : Ferdinand Brunot, fonds Brunot, méthode directe, épistémologie de l'histoire

Keywords : Ferdinand Brunot, Brunot collection, direct method, epistemology of history

\section{AUTEURS}

\section{CLÉMENTINE RUBIO}

Université de Tours, E.A. 4428 DYNADIV - clementine.rubio@univ-tours.fr 
MARC DEBONO

Université de Tours, E.A. 4428 DYNADIV - marc.debono@univ-tours.fr 